\title{
Incidence of Urinary Tract Infection, Identification of Pathogen and Drug Sensitivity Pattern in Children with Nephrotic Syndrome
}

\author{
Dr. Gobinda Mondal ${ }^{1}$, Dr. Adyasha Mohapatra ${ }^{2}$, Dr. Banasree Roy ${ }^{3 *}$, Prof. Mala Bhattacharya ${ }^{4}$ \\ ${ }^{1}$ Assistant Professor, ${ }^{2}$ DNB Ped, ${ }^{3}$ Assistant Professor, ${ }^{4}$ Principal, From Department of Paediatrics, Dr.B.C.Roy Post Graduate Institute of Paediatric \\ Sciences, Kolkata 700010, West Bengal, India
}

DOI:10.36348/SJMPS.2019.v05i09.013

| Received: 20.09.2019 | Accepted: 27.09.2019 | Published: 30.09.2019

*Corresponding author: Dr. Banasree Roy

\section{Abstract}

Introduction: Nephrotic syndrome is primarily a pediatric disorder and it affects 1-3 per 100,000 children $<16$ years of age. The prevalence of UTI is high in nephrotic syndrome patients.UTI may be responsible for poor response to steroid therapy and it also induces relapses. Objectives: To determine the incidence of urinary tract infection, identifications of pathogen and antibiotic sensitivity pattern of UTI in children with nephrotic syndrome. Method: All nephrotic syndrome patients attending the OPD or Indoor who are fulfilling the criteria were included in the study. Detailed history was taken. Routine tests including the urine culture sensitivity were sent. Result: Incidence of UTI among nephrotic syndrome patients was $18.75 \%$. E.coli, Enterococcus, Klebsiella sp., Acinetobacter was the organisms. Commonest organism found was E.coli. Aminoglycoside group of drugs found to be the most sensitive (100\%) followed by third generation cephalosporin (80\%). Conclusion: UTI is not at all uncommon in nephrotic syndrome patients and all the patients may not present with classical symptoms. Hence a high degree of suspicion is essential for early diagnosis.

Key words: Nephrotic Syndrome, Urinary tract infection, Antibiotic sensitivity.

Copyright @ 2019: This is an open-access article distributed under the terms of the Creative Commons Attribution license which permits unrestricted use, distribution, and reproduction in any medium for non-commercial use (NonCommercial, or CC-BY-NC) provided the original author and source are credited.

\section{INTRODUCTION}

Nephrotic syndrome (NS) is primarily a paediatric disorder presents with heavy proteinuria, hypoalbuminemia, hypercholesterolemia and oedema. It affects $1-3$ per 100,000 children < 16 years of age [1]. This condition is prone to several infections like urinary tract infection (UTI), cellulitis, peritonitis, septicemia, pneumonia [2]. The prevalence of UTI is high in NS and in some of the studies it has been found to be the commonest infection [3, 4]. However some studies suggest that incidence of UTI is low in the first episode and higher in subsequent relapses of NS [5].

This study was conducted to see the incidence of UTI in NS patients and to find out the etiological agents and antibiotic sensitivity pattern.

\section{Materials \& MeThoD}

This is a prospective study. The study was done in a tertiary care centre in eastern India from December 2014 to June 2016. All children with a diagnosis of nephrotic syndrome attending to the OPD were enrolled in the study. Patients with gross urogenital anomalies and already getting an antibiotic were excluded from the study.

After approval from institutional ethics committee the study was initiated. Parents of the nephrotic syndrome children fulfilling the criteria informed about the study and written consent were taken. Proper history regarding the presenting symptoms, fever, edema, urinary problems, socio economic status and family history were taken. Number of attacks or relapse was counted with the help of Bed head or OPD tickets or interviewing the attending guardian. Clinical examination was done on OPD or indoor basis. Serum albumin, urea, creatinine, routine urine analysis, USG KUB were done. A clean catch midstream urine specimen was obtained in a sterile glass tube for every patient after proper perineal cleaning and preparation. The samples were plated within one hour in Hicrome UTI Agar media and reading was taken after 48 hours. Only urine culture positive patient were included as having UTI.

All collected data was enrolled in Microsoft excel sheet and was analysed using SPSS software version 20.0.Quantitative data were analysed by mean and standard deviation and qualitative data were 
analysed by Chi square test and $\mathrm{p}$ value of $<0.05$ was taken as significant.

\section{RESUlT}

80 patients fulfilling the criteria were included in the study. Among them 15(18.75\%) had UTI. Total number of patients were distributed in 0-3years, 3.1-6 years and $>6$ years in these three age groups. Maximum number of patients i.e. 42 patients $(52.5 \%)$ fall in the 0 -3 years age group. 29 patients $(36.3 \%)$ were within 3.1-6 years and rest 9 patients $(11.3 \%)$ were $>6$ years age group but the $\mathrm{p}$ value $(0.072)$ was not significant. There were 47 male patients and 33 female patients. Among the 47 male patients 6 (12.8\%) suffered from UTI whereas 9 female patients had UTI $(27.3 \%)$.Though the incidence of UTI was higher in females but it was not statistically significant (p $>0.102)$. 47 patients $(58.8 \%)$ presented with $1^{\text {st attack. In }}$ rest of the patients $5 \%, 12.5 \%, 11.3 \%, 3.8 \%, 3.8 \%, 2.5 \%$ and $2.5 \%$ were found to be having $1^{\text {st }}, 2^{\text {nd }}, 3^{\text {rd }}, 4^{\text {th }}, 5^{\text {th }}, 6^{\text {th }}$ and $7^{\text {th }}$ relapses respectively. Only $6(12.8 \%)$ patients of $1^{\text {st }}$ attack had UTI. 50\% patients of $6^{\text {th }}$ and $7^{\text {th }}$ relapse, $66.7 \%$ patients of $4^{\text {th }}$ relapse $33.3 \%$ of $3^{\text {rd }}$ and $25 \%$ patients of $1^{\text {st }}$ relapse had UTI respectively. 22 out of 80 patients presented with fever but only $6(27.3 \%)$ turned out to have UTI. In rest of the 58 patients $9(15.5 \%)$ had UTI. But the $\mathrm{p}$ value was 0.229 so there was no significant association between fever and UTI. 4 patients presented with dysuria, 3 of them had UTI. There was a significant association between UTI and dysuria (p0.003). Among 25 patients presenting with pain abdomen 6 (24\%) had UTI whereas $16.4 \%$ of the rest 75 patients had UTI. 6 out of 80 patients had significant USG changes and 4 (66.7\%)out of them had UTI. In rest of the 74 patients without any USG changes, only $11(14.9 \%)$ had UTI. The association between USG changes and UTI was significant as the $\mathrm{p}$ value was 0.002 . Only 8 patients out of 80 had pus cell count $>5 / \mathrm{HPF}$ in urine, among them 7 had UTI $(87.5 \%)$. The $\mathrm{p}$ value was 0.0001 showing a significant association between presence of pus cell and UTI.

Out of 15 urine culture positive patients the most common organism was found to be E.coli (in 7 cases, 46.7\%). Among the other organisms Enterococcus was found in 5 cases $(33.3 \%)$, Klebsiella in 2 cases $(13.3 \%)$ and Acinetobacter boumanii in one case $(6.7 \%)$.

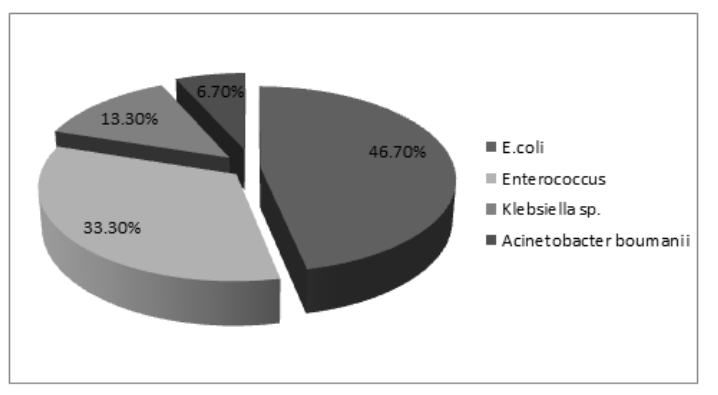

Fig-1: Distribution of microorganism as per growth in urine culture

In our study $100 \%$ of the UTI cases showed sensitivity to aminoglycosides. $3^{\text {rd }}$ generation cephalosporin were sensitive in $80 \%$ of the cases followed by sensitivity to carbapenems in $60 \%$ cases and fluroquinolones in $33.3 \%$ cases .Colistin and penicillin group of antibiotics were sensitive in $13.3 \%$ cases. Nitrofurantoin was sensitive in about $20 \%$ cases.

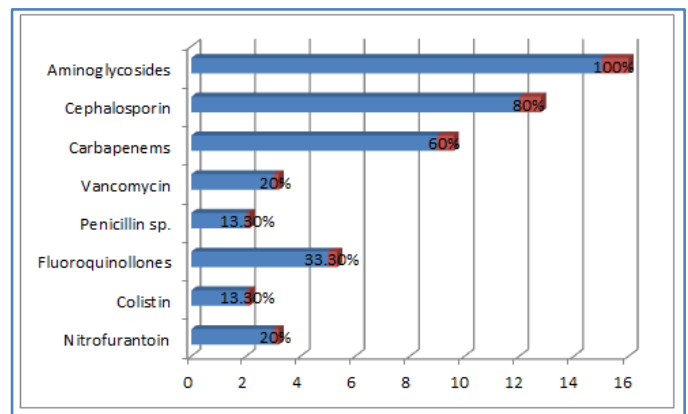

Fig-2: Distribution as per antibiotic sensitivity pattern

\section{Discussion}

The prevalence of UTI ranges from $1-3 \%$ in females and $1 \%$ in male. Beyond $1-2$ years prevalence of UTI much increases in girls and male female ratio becomes 1:10[6]. Nephrotic syndrome is an immunocompromised state due to loss of $\mathrm{IgG}$; complement B, D factor in urine. Steroid and other immunosuppressive therapies also increase the risk [7]. In our study the incidence of UTI in NS was found to be $18.75 \%$ whereas in the study by Patricia $\mathrm{Y}$ and 
others it was $46 \%$ [8]. In another study from South India the incidence was found to be $40 \%$ which are quite high than our study [5]. Lower incidence $13.8 \%$ was found in the study by Gulati et al. [4]. In our study more than half $(52.5 \%)$ patients belonged to $0-3$ year's age group. This observation was similar to the study done by Agarwal et al. [9]. The incidence of UTI was $12.8 \%$ in male and $27.3 \%$ in females in this study. In a study by Senguttuvan the incidence of UTI was $24.1 \%$ in females, slightly higher than males $22.4 \%$ [10]. On the contrary higher incidence was found in males than females in the study by Gulati et al. [4] In our study we find only $12.7 \%$ incidence of UTI among $1^{\text {st }}$ attacks and the percentage was much higher in subsequent relapses. Similar findings were seen in the study by Gulati et al. [4] In our study fever was not a very consistent symptom associated with UTI as only $27.3 \%$ patients with fever had UTI whereas $13.2 \%$ develop UTI among the afebrile group. Dysuria had a significant association with UTI in this study $(\mathrm{p}<0.003)$. In our study among 25 patients presenting with pain abdomen $6(24 \%)$ had UTI. Similarly Sreenivas et al. reported fever in $18 \%$ cases, dysuria in $8 \%$ cases and abdominal pain in $10 \%$ patients of UTI as a presenting symptom [5] Only in 10\% cases urine microscopy revealed presence of pus cell $>5 / \mathrm{hpf}$ in our study and among them $7(87.5 \%)$ had UTI ( 1 <0.05).In rest of the patients 8 turned out to be positive growth in urine culture. So urine RE ME cannot be used alone to diagnose UTI. This finding further supported in various studies by Gulati et al. Sreenivasa et al. [4, 5]. In this study 6 patients found to have certain changes on USG KUB and 4 out of them had UTI which was statistically significant $(\mathrm{p}<0.002)$.

The most common organism detected in our study was E.coli (46.7\%) followed by Enterococcus $(33.3 \%)$ and Klebsiella sp (13.3\%). E.coli was found to be the predominant organism causing UTI in various studies like Gulati et al. (61\%), Sreenivasa et al. $(50 \%)[4,5]$. In another study by Adeleke et al. Staphylococcus was the most common pathogen followed by Kebsiella[3]. We observed high sensitivity to Aminoglycosides (100\%) and up to $80 \%$ sensitivity for $3^{\text {rd }}$ and $4^{\text {th }}$ generation Cephalosporins like Ceftriaxone, Cefotaxime, Cefaperazone sulbactum, Cefpodoxime, Cefepime and cefepime tazobactum. About $60 \%$ sensitivity was observed in Carbapenems such as Imipenem, Imipenemcillastatin, Meropenem. On the other hand Fluoroquinolones were found to be sensitive in only $33.3 \%$ cases. Antibiotics like Penicillin group, Vancomycin, Nitrofurantoin had lower sensitivity in the present study. Similar antibiotic susceptibility was found in the study by Sreenivasa et $a l$. Where majority of the organisms were found to be sensitive to $3^{\text {rd }}$ generation Cephalosporins and Aminoglycosides [5]. In the study by Patricia Y.Gunawan and Adrian Umboh E. coli was one of the common organisms and Imipenem and Amikacin were found to be most common sensitive antibiotics[8].

\section{Conclusion}

UTI is not at all uncommon among children suffering from nephrotic syndrome. Just a routine microscopic examination may not be sufficient for the diagnosis of UTI .All patients may not present with classical symptoms of UTI. So always culture sensitivity must be done. Though E. coli is responsible for majority of cases but non E.coli organisms are not very infrequent.

\section{REFERENCE}

1. Pais. P., Avner E.D. Nephrotic Syndrome. In: Kliegman R.M., Santon B.F., St. Geme J.W., Schor N.F. (2015). Editors. Nelson Textbook of Pediatrics. First South Asia Edition. India: Elsevier, (2), 2521-7.

2. WEI, C. C., YU, I. W., LIN, H. W., \& Tsai, A. C. (2012). Occurrence of infection among children with nephrotic syndrome during hospitalizations. Nephrology, 17(8), 681-688.

3. Adeleke, S. I., \& Asani, M. O. (2009). Urinary tract infection in children with nephrotic syndrome in Kano, Nigeria. Annals of African medicine, 8(1), 38.

4. Gulati, S., Kher, V., Arora, P., Gupta, S., \& Kale, S. (1996). Urinary tract infection in nephrotic syndrome. The Pediatric infectious disease journal, 15(3), 237-240.

5. Sreenivasa, B., Murthy, C. S., Raghavendra, K., Basavanthappa, S., Pejaver, R., \& Jadala, H. V. (2015). Urinary tract infection at presentation of nephrotic syndrome: A clinical evaluation. Indian J Child Health, 2(1), 1-4.

6. Jack, S., Elder. Urinary Tract Infection. (2015). In: Kliegman R.M., Santon B.F., St. Geme J.W., Schor N.F. editors. Nelson Textbook of Pediatrics. First South Asia Edition. India: Elsevier, 2, 2556.

7. Noer, M. S. Sindrom nefrotik idiopatik. In: Noer MS, Soemyarso NA, Subandiyah K,Prasetyo RV,Atlas H, Tambunan T, (2011). Editors. Kompendium nefrologi anak.Jakarta: Badan Penerbit Ikatan Dokter Anak Indonesia, 72-87.

8. Gunawan, P. Y., \& Umboh, A. (2016). The risk of urinary tract infection in children with nephrotic syndrome. Paediatrica Indonesiana, 56(4), 238-41.

9. Chowdhury, V.P., Agarwal, R. (1977). Urinary tract infection in children. Indian Pediatr, 14:849853.

10. Senguttuvan, P., Ravanan, K., Prabhu, N., \& Tamilarasi, V. (2004). Infections encountered in childhood nephrotics in a pediatric renal unit. Indian J Nephrol, 14(3), 85-88. 